\title{
Du naturalisme au naturisme : mutations idéaliste et identitaire à travers l'oeuvre du peintre belge Léon Frederic (1856-1940)
}

\section{Benjamin Foudral}

Volume 41, numéro 2, 2016

The Nature of Naturalism : A Trans-Historical Examination

La nature du naturalisme : un examen transhistorique

URI : https://id.erudit.org/iderudit/1038072ar

DOI : https://doi.org/10.7202/1038072ar

Aller au sommaire du numéro

Éditeur(s)

UAAC-AAUC (University Art Association of Canada | Association d'art des universités du Canada)

ISSN

0315-9906 (imprimé)

1918-4778 (numérique)

Découvrir la revue

Citer cet article

Foudral, B. (2016). Du naturalisme au naturisme : mutations idéaliste et identitaire à travers l'oeuvre du peintre belge Léon Frederic (1856-1940). RACAR : Revue d'art canadienne / Canadian Art Review, 41(2), 62-76. https://doi.org/10.7202/1038072ar

\section{Résumé de l'article}

In an article published in 1895 in the Belgian periodical L'Art Jeune, the writer and art critic Henri Vandeputte introduced the artist Léon Frederic - whom he called "le Premier des peintres belges"- as the leading figure of a new artistic neologism : naturisme. This is one instance that points to the consistent critical extrapolation and ideological exploitation of Frederic and his unclassifiable paintings. This article focuses on the reception of Frederic's work in order to understand how artistic terms such as réalisme, naturalisme, and naturisme were used in the name of a nationalist art history in which Belgian art could both claim the legacy of Flemish art and detach itself from the dominant model of French art
Tous droits réservés (C) UAAC-AAUC (University Art Association of Canada | Association d'art des universités du Canada), 2016
Ce document est protégé par la loi sur le droit d'auteur. L'utilisation des services d’Érudit (y compris la reproduction) est assujettie à sa politique d'utilisation que vous pouvez consulter en ligne.

https://apropos.erudit.org/fr/usagers/politique-dutilisation/ 


\title{
Du naturalisme au naturisme: mutations idéaliste et identitaire à travers l'œuvre du peintre belge Léon Frederic (1856-1940)
}

\author{
Benjamin Foudral
}

\begin{abstract}
In an article published in 1895 in the Belgian periodical L'Art Jeune, the writer and art critic Henri Vandeputte introduced the artist Léon Frederic-whom he called "le Premier des peintres belges"-as the leading figure of a new artistic neologism: naturisme. This is one instance that points to the consistent critical extrapolation and ideological exploitation of Frederic and his unclassifiable paintings. This article focuses on the reception of Frederic's work in order to understand how artistic terms such as réalisme, naturalisme, and naturisme were used in the name of a nationalist art history in which Belgian art could both claim the legacy of Flemish art and detach itself from the dominant model of French art.
\end{abstract}

Benjamin Foudral est doctorant contractuel en histoire de l'artà I'Université Paris-Sorbonne (Paris IV) -ben.foudral@gmail.com

1. Bien que le nom de l'artiste soit orthographié «Frédéric» dans la plupart des textes ou au sein des institutions, nous préférons adopter le nom de Frederic tel qu'il est officiellement inscrit dans les registres d'état civil et tel que Léon Frederic l'utilisait lui-même pour signer.

2. Henri Vandeputte, «Léon Frédéric», L'ArtJeune, Bruxelles, $n^{\circ} 5$, 15 mai 1895, p. 107.

3. Gilles Candar, «Belgique et France 1848-1914", dans ParisBruxelles. Bruxelles-Paris, catalogue d'exposition, Paris et Gand, 1997, p. 26-34.
Dans un article paru en 1895 dans la revue L'Art jeune, Henri Vandeputte, écrivain et critique d'art, intronise l'artiste Léon Frederic, ${ }^{1}$ «le Premier des peintres belges», comme chef de file d'un énième néologisme artistique: le naturisme. ${ }^{2}$ Peintre développant une philosophie chrétienne proche du panthéisme, Frederic-tout comme son œuvre inclassable-n'a cessé d'être sujet aux extrapolations critiques et aux récupérations idéologiques, si courantes en histoire de l'art. La présente contribution souhaite interroger, à travers l'œuvre de Frederic et de ses contemporains, un phénomène esthétique, celui de l'émergence d'un naturalisme international et de sa rapide mutation en Belgique vers une voie idéaliste, nommée par certains le naturisme. Ces termes seront conjointement disséqués au regard du discours des premiers historiens de l'art et de la critique d'art de l'époque, que l'on verra tourné vers le nationalisme et la recherche d'une identité propre. Il s'agira de comprendre dans quelles mesures certains termes artistiques-ici, le réalisme, le naturalisme puis le naturisme-bien que représentant des moments artistiques identifiables et identifiés, ont pu servir dès leur apparition de fer de lance idéologique et politique à la construction d'une histoire de l'art orientée.

\section{La jeune génération artistique belge face au sentiment national}

Née en 1830 et radicalement transformée par les nombreuses réformes industrielles du règne de Léopold $\mathrm{II}$, la Belgique parvient à rivaliser d'un point de vue économique avec les autres puissances européennes et se positionne comme un exemple de modernité et de progrès. ${ }^{3}$ Prenant ses distances vis-àvis des diverses tutelles étrangères qui ont scandé son histoire, ce pays s'est ainsi retrouvé en proie à de profondes interrogations identitaires.

Dans un besoin de légitimation politique, les autorités réfléchissent dès 1830 à pallier le manque de continuité ethnique et historique de ce territoire par la culture. Comme l'analyse très justement Laurence Brogniez, la "peinture constitue un enjeu stratégique pour les autorités politiques soucieuses de légitimer l'existence du jeune État [...]». ${ }^{4}$ Les «ancêtres picturaux» universellement réputés tels que Pierre-Paul Rubens, Jacob Jordaens et Antoine van Dyck sont alors présentés comme les meilleurs représentants d'un héritage commun. La peinture fournira ainsi des signes identitaires forts et susceptibles de combler ce que Christian Berg nomme un «déficit symbolique», ${ }^{5}$ tout en affirmant la singularité de ces glorieux ancêtres et en misant sur leur reconnaissance à l'étranger. La quête identitaire belge prend un nouvel essor dès 
4. Laurence Brogniez, «Nés peintres: la "prédestination merveilleuse" des écrivains belges», dans La Belgique entre deux siècles: Laboratoire de la Modernité, 1880-1914, Berne, 2007, p. 88.

5. Christian Berg, «Le déficit symbolique: la littérature française face au mouvement flamand en Belgique au XIX ${ }^{\mathrm{e}}$ siècle», dans Literatur im französischsprachigen Belgien. Akten der Belgiensektion des Deutschen Romanistentages Aachen, Berne et Paris, 1990 p. $161-172$.

6. F.-G. Dumas, 1830-1880. Ca talogue illustré de l'Exposition historique del'Art belge et du Musée moderne de Bruxelles, Bruxelles, 1880, p. 4.

7. Sébastien Clerbois, L'Ésotérisme et le symbolisme belge, Wijnegem, 2012, p. 146.

8. Éric Michaud, Les Invasions barbares. Une généalogie de l'histoire de l'art, Paris, 2015, p. 11-26, ici p. 16.

9. Brogniez, op. cit., p. 90

10. Camille Lemonnier et Adolphe Samuel, Cinquante ans de liberté, tome III: Histoire des beaux-arts en Belgique (1830-1887), $2^{\mathrm{e}}$ éd., Bruxelles, 1887, p. 303.

11. Camille Lemonnier et AdoIphe Samuel, Cinquante ans de liberté, tome III: Histoire des beaux-arts en Belgique, Bruxelles, 1881, p. 7.
1880 à l'occasion du cinquantenaire de l'indépendance du pays et de l'Exposition historique de l'Art belge 1830-1880. Cette manifestation «exclusivement nationale» souhaitait «dresser le bilan de [la] production artistique pendant un demi-siècle et mesurer le chemin parcouru» afin de démontrer que les nouvelles générations faisaient jeu égal avec un «passé [qui] occup[ait] une place si considérable dans l'histoire de l'art». ${ }^{6}$ Devant le constat, observé lors de cette exposition et des expositions universelles, d'une école belge irriguée par les innovations esthétiques venues de l'étranger, la problématique culturelle et identitaire ressurgit. La référence aux maîtres de la peinture tels que Rubens n'ayant pas suffi à créer cette identité commune tant recherchée, les intellectuels belges ont recherché un concept capable d'une part de cerner l'atavisme artistique d'un pays traversé par des courants esthétiques aussi divers que contradictoires et d'autre part de servir de grille de lecture à l'analyse de l'art belge. Comme le rappelle Sébastien Clerbois, c'est «à cette fin que les critiques d'art conçoivent ce qui est appelé l'art flamand ". ${ }^{7}$ Ce terme, à l'époque strictement culturel, comptait englober les diverses expressions artistiques autour d'un dénominateur commun rattaché à la tradition picturale du pays prenant sa source chez les Primitifs flamands du xve siècle et se poursuivant aux époques postérieures: le réalisme. Ce «fantasme de filiation» est constitutif du discours de l'histoire de l'art, un discours qui, comme le démontre Éric Michaud, a voulu depuis ses débuts se «donn[er] pour tâche de décrire les objets produits par des peuples présumés homogènes et demeurant de siècle en siècle toujours identiques à eux-mêmes». ${ }^{8}$ L'Europe entière est en proie aux réflexions ethniques, voire racialistes, contribuant à la construction des États-nations, et la Belgique n'est pas à l'abri de ce phénomène. Le territoire belge devient ainsi le berceau géographique qui aurait vu naître des artistes et des objets qui seraient le témoignage irréfutable d'une identité et d'une homogénéité fantasmées d'un peuple qui a acquis son indépendance.

L'un des initiateurs de cette nouvelle histoire de l'art belge est l'écrivain Camille Lemonnier, qui jouissait à cette époque d'une aura internationale importante grâce à son entrée comme jeune critique d'art sur la scène européenne avec sa biographie de Gustave Courbet parue en 1868 et son ouvrage intitulé Les Flamands publié l'année suivante. Ce dernier, un recueil d'essais consacré à l'art national et aux mœurs de la Belgique, servira de fondement à l'édifice que veut construire Lemonnier, celui d'une théorie cohérente de l'art "procédant d'une lecture ethnique de la peinture, de sa forme comme de ses sujets». ${ }^{9}$ Comme en témoignera plus tard Lemonnier, l'Exposition historique de 1880 devient alors le catalyseur de l'histoire de l'art belge et une nouvelle étape dans sa construction intellectuelle:

L'Exposition historique de 1880 fut une date dans l'histoire des arts en Belgique: elle condensa, dans une remarquable impression d'ensemble, comme la synthèse et la succession des tentatives de plusieurs générations d'artistes pour arriver à une formule rationnelle et conforme au tempérament de la race. ${ }^{10}$

Encouragé par les célébrations du cinquantenaire de l'indépendance, Lemonnier publie en 1881 un ouvrage fondateur, Histoire des Beaux-arts en Belgique, qui est le troisième tome d'un vaste ouvrage intitulé Cinquante ans de liberté et qui traite de l'évolution de la scène artistique belge depuis l'indépendance du pays. Partant du constat que «la vieille tradition flamande», ${ }^{11}$ définie par 
2. Ibid., p. 12.

3. Ibid., p. 15.

14. Paul Gorceix, «Camille Lemonnier ou l'identité picturale belge», Bulletin de l'Académie royale de langue et de littérature française, tome LXXII, 1-2, 1994, p. 269-281.

15. Les cours dispensés par Taine à l'École des beaux-arts ont été progressivement édités puis réunis sous le titre de Philosophie de l'art, dont le premier volume parut en 1865 .

16. Angèle Kremer-Marietti, "Sur l'esthétique de Taine», Romantisme, vol. 11, n³2, 1981, p. 23-29.

17. Yves Michaud, «L'Artiste: dans son temps ou vu par le nôtre», dans Matthias Waschek (dir.), Relire Taine, Paris, 2001, p. 81-100.

18. Lemonnier, Histoire des beaux-arts en Belgique, op. cit., 1881, p. 112.

19. Ibid., p. 179

20. Ibid. l'observation de la réalité, «l'outrance de la couleur et [la] bonhomie dans la composition", ${ }^{12}$ et trouvant son apogée dans l'art de Rubens, se mourait, Lemonnier accuse l'école néoclassique et l'exil du peintre français JacquesLouis David en Belgique de lui avoir donné le coup de grâce: «Au lieu de développer chez les Flamands la tendance native parallèlement à l'observation de la réalité, [David] acheva de l'absorber dans son effrayante individualité. Là fut son erreur; là fut son crime». ${ }^{13}$ Les cinquante années qui ont suivi l'arrivée de David sont analysées à travers un même prisme: la quête de la tradition flamande dans l'école belge. L'analyse séduisante d'un art belge basé sur l'observation de la réalité et la prédominance de la matière et du coloris sur le dessin, soit le concept de la picturalité, stimule les critiques d'art et devient rapidement une grille de lecture pour une majorité d'observateurs. ${ }^{14}$ Ce raisonnement n'est pas le seul fait de Lemonnier. L'auteur belge se place dans la lignée tracée depuis le début des années 1860 par Hippolyte Taine, successeur d'Eugène Viollet-le-Duc à l'École des beaux-arts de Paris, où il assurait un cours sur l'enseignement de l'histoire de l'art et l'esthétique. Taine développa une philosophie de l'art qui rend compte des œuvres comme produits des civilisations qui les voient naître. ${ }^{15}$ La méthode comparatiste de Taine distingue ainsi trois facteurs essentiels-la race, le milieu et le moment-ce qui la conduit à une forme de déterminisme de la production artistique. ${ }^{16}$ Pour Taine, plus un poète/artiste est national, plus il est parfait. ${ }^{17}$ Les ouvrages et la pensée systémique de Taine ont irrigué de nombreux pays européens dont la Belgique, où la réception en était favorisée par le partage d'une même communauté de langue et par la publication en 1869 d'un ouvrage, La Philosophie de l'art des PaysBas, qui concernait aussi bien la Hollande que la Belgique. L'idée d'une correspondance entre l'œuvre, le pays et le siècle a également séduit les historiens de l'art belge, notamment Lemonnier, qui applique la vision tainienne et justifie ainsi le réalisme qu'il identifie comme le dénominateur commun de l'art belge et inhérent au "peintre de race» belge. Malgré les timides inclinaisons réalistes observées par Lemonnier chez quelques artistes de la première moitié du XIXe siècle comme François-Joseph Navez, le critique loue le rôle de Gustave Courbet, déjà développé dans l'ouvrage qu'il avait consacré au maître, et l'exposition de ses Casseurs de pierres lors du Salon bruxellois de 1851, pour l'impulsion considérable que cet artiste a eue sur l'école belge. ${ }^{18}$ Lemonnier ne souhaite toutefois pas introniser l'artiste français comme initiateur de la nouvelle école nationale, et accorde donc cette place aux peintres belges Charles de Groux et Henri Leys. Ce dernier devient la personnalité phare d'une nouvelle tendance, celle d'un renouveau de l'art national «basé sur une conception réaliste, avec l'emploi des formules qui, de tout temps, ont été le mieux appropriées au génie de la race [belge]». ${ }^{19}$ Dès lors, l'expression de la nature qui est encore timide au sein de l'Académie devient, pour Lemonnier, «synthétique et naturaliste» grâce à «la contemplation immédiate de l'homme dans son milieu». ${ }^{20}$ Cette nouvelle aspiration véhiculée par de nombreux artistes belges a déterminé la création en 1868 d'un cercle d'artistes dont il était l'un des fondateurs, la Société libre des Beaux-arts. L'école belge retrouve ainsi, selon Lemonnier, la voie qui correspond le mieux au tempérament de son peuple. L'édition augmentée et corrigée de l'Histoire des beaux-arts en Belgique parue en 1887 n'est qu'un moyen pour lui de démontrer la vitalité 
21. Lemonnier, Histoire des beaux-arts en Belgique, $2^{\mathrm{e}}$ ed., op. cit. 1887, p. 311.

22. Il s'agirait, comme nous le retrouvons sous la plume du peintre français Henri Testelin (1616-1695), de «l'imitation exacte du naturel en toutes choses». Pour de plus amples informations concernant le succès du terme «naturalisme» au XVII ${ }^{\mathrm{e}}$ siècle voir Christian Michel, «Le Chevalier Bernin et l'enseignement artistique en France», dans Chantal Grell et Milovan Stanic (dir.), Le Bernin et l'Europe, Paris, 2002, p. 95-105

23. Pour plus de précisions voir Richard Thomson, Art of the Actual: Naturalism and Style in Early Third Republic France, 1880-1900, Londres, 2012.

24. Jules-Antoine Castagnary, Salons. 1857-1870, Paris, 1892, p. 100-182. L'article consacré au Salon de 1863 a été publié dans la revue bruxelloise Nord, les 14, 19, 27 mai, 4 juin, $1^{\text {er }}$ et 15 août et 12 septembre 1863 .

25. La Rédaction, «Notre Programme», L'Artiste, Bruxelles, 1 ère année, $n^{\circ} 51,24$ décembre 1876 , p. $415-416$.

26. Camille Lemonnier, Gustave Courbet et son œuvre, Paris, 1868 , p. 36. de la nouvelle génération de 1880 et son attachement à la nouvelle voie qu'il a identifiée, notamment à travers les œuvres de Frans Courtens, Jacques de Lalaing, Charles Goethals, Léon Abry, James Ensor, Guillaumes Vogels, Léon Frederic, et autres: celle du naturalisme. ${ }^{21}$

Employé dans l'acception donnée depuis le xvil ${ }^{\mathrm{e}}$ siècle $^{22}$ et comme synonyme de réalisme par Lemonnier, le terme de naturalisme reste discret tout au long de l'Histoire des Beaux-arts en Belgique. Depuis les années 1860, ce vocable évolue et intervient dans la définition d'un nouveau courant pictural puis littéraire qui s'inscrit dans la continuité du réalisme des années 1850. Les décennies 1860-1870 jouent un rôle considérable dans l'éclosion de ce nouveau mouvement grâce au contexte politique, social, économique ${ }^{23}$ et scientifique, comme l'affirme le critique d'art Jules-Antoine Castagnary dans son Salon de 1863. Dans cet article manifeste, Castagnary définit le naturalisme comme «l'expression de la vie sous tous ses modes et à tous ses degrés» et dont le "but unique est de reproduire la nature en l'amenant à son maximum de puissance et d'intensité: c'est la vérité s'équilibrant avec la science». ${ }^{24}$ Depuis le début des années 1870 , Lemonnier est l'une des figures majeures de ce mouvement, d'abord en tant que défenseur du naturalisme pictural, puis comme principal représentant du naturalisme littéraire en Belgique. C'est en 1877 que ce mouvement s'implante avec force dans ce pays, grâce en partie au poète et artiste Théodore Hannon, qui s'impose en tant que rédacteur en chef de L'Artiste, fondée à Bruxelles deux ans plus tôt. Hannon donne une impulsion nouvelle à cette revue jusque-là modérée dans la défense des nouvelles tendances. Il y prône le naturalisme comme la seule voie de la modernité, définie comme «le culte ému, la mystérieuse intuition de la Nature» et «la liberté individuelle en matière d'art». ${ }^{25}$ Lemonnier anime, quant à lui, L'Art universel depuis $1873 \mathrm{et}$ L'Actualité à travers le monde et l'art depuis 1876, qui devient peu à peu un organe du courant naturaliste, mais qui cessera de paraître en août 1877 . Auréolé de sa reconnaissance internationale, en particulier à Paris, Lemonnier rejoint alors L'Artiste et y poursuit la défense du naturalisme, qu'il avait défini dès 1868 dans son ouvrage consacré à Courbet:

Le naturalisme est le réalisme agrandi de l'étude profonde des milieux et de l'observation nette des caractères. Le naturalisme suppose une philosophie que n'avait pas le réalisme, et en effet c'est toute une philosophie qui par ses bouts tient à la biologie, à la géologie, à l'anthropologie, aux sciences exactes et aux sciences sociales. Le naturalisme en art est la recherche du caractère par le style, de la condition par le caractère, de la vie entière par la condition; il procède de l'individu au type et de l'unité à la collectivité. ${ }^{26}$

Lemonnier fait ici référence au positivisme de Taine appliqué à l'art, mais aussi à l'article sur le naturalisme de 1863 de Castagnary. Pour Lemonnier, du moins à cette époque, le positivisme est la source du naturalisme.

Grand défenseur de la nouvelle voie naturaliste depuis le début des années 1870 , Lemonnier en est néanmoins un soutien timoré dans son ouvrage phare L'Histoire des Beaux-arts de 1881, notamment à cause de la disparition du terme de naturalisme dans l'acception qu'il en donnait depuis 1868. Ce revirement intellectuel semble trouver une explication dans le nationalisme revendiqué par Lemonnier. Dès la fin des années 1870, le naturalisme pictural fait une entrée fracassante dans les différentes expositions européennes 
27. Béatrice Joyeux-Prunel, $\mathrm{Nul}$ n'est prophète en son pays? L'internationalisation de la peinture des avant-gardes parisiennes, 1855-1914, Paris, 2009, p. 44-46; Robert Jensen, Marketing Modernism in Fin-de-siècle Europe, Princeton, 1994, p. 138-166.

28. Lucien Solvay, L'Art et la liberté, Bruxelles, 1881, p. 23. L'auteur fonde sa théorie esthétique, tout comme Taine et Lemonnier, sur l'accord entre l'art et le sentiment national.

29. Ibid., p. 45.

30. Ibid., p. 78-79.

31. Ibid., p. 233

32. Ibid., p. 239-241. et en particulier en Belgique grâce à l'école française et notamment aux œuvres du jeune Jules Bastien-Lepage. Décrit par les historiens de l'art d'aujourd'hui comme le peintre du «juste milieu» ${ }^{27}$ entre académisme et modernité, Bastien-Lepage reste l'une des figures artistiques fondamentales dans l'émergence d'un naturalisme international, ayant recueilli les suffrages des critiques les plus divers, en France comme à l'étranger. Après l'impulsion donnée par le réalisme de Courbet à l'école belge, cette nouvelle suprématie française ne pouvait être défendue par Lemonnier, car elle aurait signé l'échec de son entreprise personnelle, celle de donner une identité propre à l'art belge. Si le naturalisme perd ainsi un partisan de choix, il en retrouve un autre sous la plume de Lucien Solvay, dont l'ouvrage L'Art et la Liberté paru en 1881 reprend le postulat de Lemonnier concernant «le sentiment national dans les Arts». ${ }^{28}$ Tout en reconnaissant que la France et ses artistes ont eu au $\mathrm{xIX}^{\mathrm{e}}$ siècle un impact indéniable sur l'art belge, Solvay interprète les innovations françaises comme ayant été inspirées par l'art flamand:

C'est de la France, on ne saurait le nier, que sont parties toutes les grandes rénovations artistiques de ces derniers temps. [...] Cependant, si aujourd'hui la nature, grâce à elle, affirme dans l'art sa puissance longtemps méconnue, il est juste de dire que c'est en portant ses regards vers le Nord, en se souvenant de l'art flamand et hollandais du XvII ${ }^{\mathrm{e}}$ siècle, que la France a pu concevoir l'idée bienfaisante de ce retour à la vérité et à la vie. ${ }^{29}$

La voie des nouvelles générations d'artistes belges est ainsi toute tracée par ces critiques, s'inscrivant dans les pas des artistes réalistes prônant un art "sous l'inspiration directe de la nature, et ramené ainsi peu à peu aux sources pures du vieil art flamand».30 Toutefois, contrairement à Lemonnier qui utilisait les termes «réalisme » et «naturalisme» sans distinction, Solvay clôt son ouvrage par une étude précise du naturalisme artistique qu'il considère comme le stade ultime de la modernité à laquelle doit aspirer l'école belge. Pour lui, le naturalisme est en parfait accord avec la tradition réaliste flamande, puisqu'il la perpétue et la complète par les apports de la science et l'observation stricte du quotidien. Reprenant les idées de Castagnary, le naturalisme serait l' '«abolition des poncifs et des recettes d'écoles; [...] l'art libre, dégagé des lisières académiques et ne relevant que de lui-même». ${ }^{31}$ À l'opposé de Lemonnier, Solvay ne semble pas se soucier des influences étrangères et n'hésite pas à citer en guise de conclusion Bastien-Lepage, qui devient sous sa plume le chef de file du naturalisme. ${ }^{32}$

La jeune génération d'artistes belges de 1880 va s'engager dans cette nouvelle voie défendue par la critique progressiste. C'est le cas du cercle artistique L'Essor fondé en 1879-continuité du Cercle des anciens élèves et élèves des Académies des beaux-arts de Bruxelles-qui souhaitait par ses propres expositions défendre les innovations artistiques rejetées par l'Académie et les jurys des salons officiels. Léon Frederic adhère à L'Essor dès 1880, après son voyage de formation en Italie. Fasciné par l'exposition des Foins (1877) de BastienLepage au Salon de Bruxelles en 1881, Frederic se détourne brusquement de l'art académique de son professeur Jean-François Portaels qui, malgré un enseignement libéral, préférait les compositions historiques et religieuses aux représentations de la vie quotidienne prônées par les défenseurs du naturalisme. Dès 1881, Frederic passe d'une peinture d'atelier composée de scènes ou de portraits représentés dans un décor d'intérieur reconstitué de toutes 


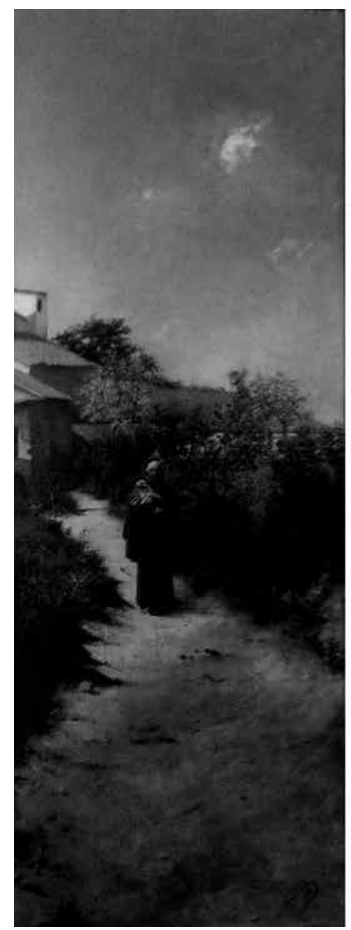

Figure 1. Léon Frederic, La Légende de saint François, 1882, huile sur toile, $136 \times 100 \mathrm{~cm}$ (panneau central); $136 \times 51 \mathrm{~cm}$ (panneaux latéraux). Bruxelles, collection privée (photo de l'auteur).

33. Ed. Isart, «Lettre sur le SaIon d'Anvers. Deuxième», Journal des Beaux-arts et de la littérature, Bruxelles, $24^{\mathrm{e}}$ année, $\mathrm{n}^{\circ} 16,31$ août 1882 , p. 123-125.

34. Alphonse Wauters, «Le Salon d'Anvers", L'Echo du parlement, Bruxelles, $25^{\mathrm{e}}$ année, $\mathrm{n}^{\circ} 230,18$ août 1882, p. 2; Emile Verhaeren, "Exposition du Cercle artistique», Journal des Beaux-arts et de la littérature, Bruxelles, $24^{\mathrm{e}}$ année, $n^{\circ} 8,30$ avril 1882 , p. 57-58.

35. Anonyme, «Exposition du Cercle artistique de Bruxelles. Troisième article», L'Art moderne, Bruxelles, $2^{\mathrm{e}}$ année, $\mathrm{n}^{\circ} 18,30$ avril 1882, p. 137.

36. Lettre autographe et signée de Bernard Scié à Léon Frederic, datée du 1 er janvier 1891 ; L.A.S. de Bernard Scié à Léon Frederic, datée du $1^{\mathrm{er}}$ janvier 1892. Folio Documentation S.v 82641 et $82642-$ Cabinet des Estampes de la Bibliothèque Royale de Belgique, Bruxelles.
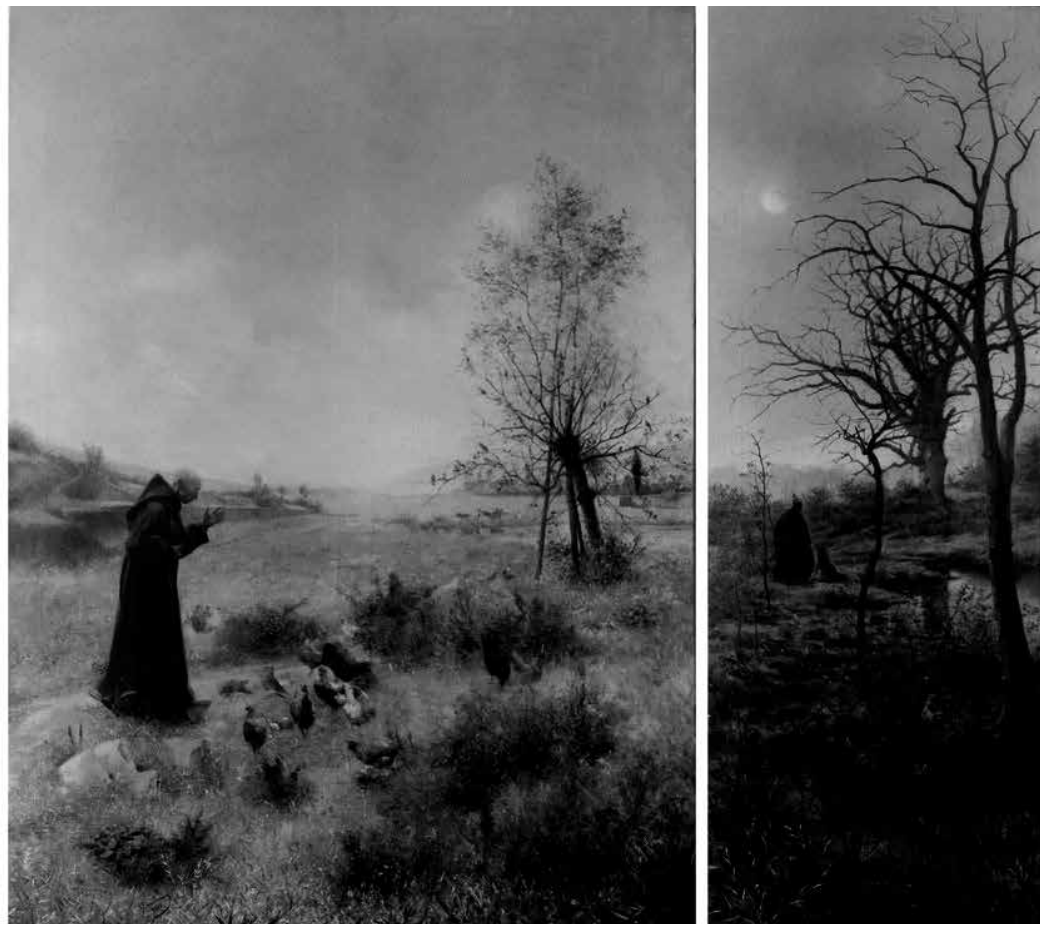

pièces à l'application d'une formule dite naturaliste et proche de l'art de Bastien-Lepage. Cette formule souhaite affirmer l'importance de l'étude d'après nature et du plein air. La première œuvre manifestant en partie la nouvelle manière de Frederic s'intitule La Légende de saint François (1881). | fig. 1 | Le tableau représente, sous la forme d'un triptyque, le Poverello dans trois scènes de plein air, illustrant trois temps de la journée, trois saisons et trois scènes de la vie du saint. Les critiques de l'époque ont soulevé, non à tort, le souvenir d'Ernest Duez et de son triptyque, Saint Cuthbert, qui remporta un vif succès au Salon de 1879 et fut acquis par l'État français la même année. ${ }^{33}$ Les deux œuvres sont comparables par le retour à la forme du triptyque et l'insertion du personnage dans un paysage. L'originalité de Frederic tient au traitement pleinairiste du paysage et au choix de la figure du saint. Le peintre a choisi intentionnellement de délaisser les modèles traditionnels d'atelier pour leur préférer une personne humble, un déshérité du nom de Bernard Scié, marchand de craie habitant Saint-Gilles. L'artiste semble ainsi avoir voulu faire correspondre la condition sociale de son modèle à la condition de saint François, l'époux de «Dame pauvreté». L'œuvre a rencontré un succès critique certain auprès de l'ensemble des revues et quotidiens de l'époque ${ }^{34}$ qui, malgré quelques remarques négatives concernant le traitement de la couleur, ont intronisé Frederic comme «[l']une des espérances de l'école [belge]» et de "l'école naturaliste». ${ }^{35}$ Comme en atteste une correspondance lacunaire, Frederic s'est lié d'amitié avec Scié, ${ }^{36}$ qui lui a décrit, lors des séances de pose, les conditions difficiles de son quotidien de marchand de craie. Ce récit est en quelque sorte une révélation pour l'artiste qui évoquera, dès lors, à de 


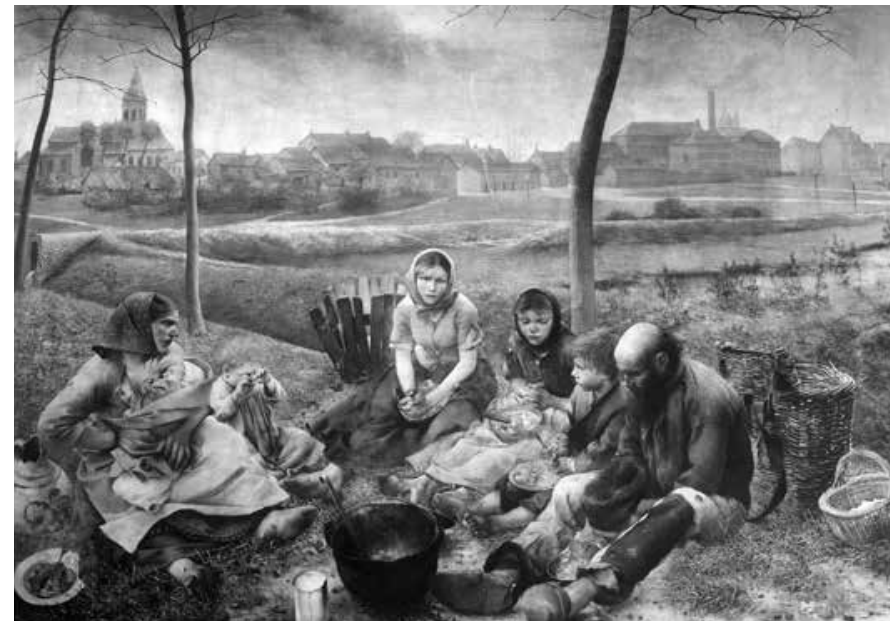

Figure 2. Léon Frederic, Le Midi, 1882, huile sur toile, marouflée sur bois, panneau central du triptyque Les Marchands de craie, $1882-1883,200 \times 268 \mathrm{~cm}$. Bruxelles, Musées royaux des Beaux-arts de Belgique (photo (c) KIK-IRPA, Bruxelles, Belgique).

37. Herwig Todts, «Le naturalisme: introduction à l'historiographie et à l'interprétation d'une mode», dans Tranches de vie. Le naturalisme en Europe, catalogue d'exposition, Anvers, Musées royaux des Beaux-arts, 1996, p. 9-31.

38. Lemonnier, Histoire des Beaux-arts en Belgique, $2^{\mathrm{e}}$ ed., op. cit., 1887, p. 319.

39. Emile Verhaeren, «Chronique artistique», La Jeune Belgique, Bruxelles, $3^{\mathrm{e}}$ année, $n^{\circ} 6,28$ avril 1883, p. 232.

40. Pierre Gervaix, «Le Salon de Gand (Suite)", Journal des Beauxarts et de la littérature, Bruxelles, $25^{\circ}$ année, $n^{\circ} 18$, 30 septembre 1883 , p. 139.

41. Anonyme, «Le Salon de Bruxelles», L'Art moderne, Bruxelles, $1^{\text {ère }}$ année, $n^{\circ} 33,16$ octobre 1881 p. 250.

42. Emile Verhaeren, «Exposition du Cercle artistique», Journal des Beaux-arts et de la littérature, Bruxelles, $24^{\mathrm{e}}$ année, $n^{\circ} 8,30$ avril 1882, p. 57-58.

43. Ibid. multiples reprises dans ses œuvres le sort de ces déshérités dans la veine du naturalisme social que l'on retrouve dans son œuvre phare Les Marchands de craie (188). ${ }^{37}$ | fig. 2 | Le peintre y reprend la même facture et le même format que dans sa Légende de saint François. Cependant, le sujet religieux disparaît pour laisser place à la représentation de trois moments de la vie d'une famille de marchands de craie en banlieue bruxelloise. Selon plusieurs critiques, l'œuvre trahissait encore une fois, tant par la composition que par le coloris et la facture, l'influence de Bastien-Lepage. ${ }^{38}$

Le milieu artistique belge, autant la critique que les artistes eux-mêmes, a dans un premier temps perçu favorablement les œuvres de Bastien-Lepage. L'inclinaison de ce dernier vers la représentation fidèle du quotidien des humbles et l'observation directe de la nature ne faisaient que renforcer cette réception positive. Pourtant assez rapidement, comme le souligne la sentence du poète et critique d'art Emile Verhaeren, l'impact de son art est devenu un problème: «qui nous débarrassera, je ne dis pas de l'influence, mais de l'imitation de Bastien-Lepage?». ${ }^{39}$ De nombreux critiques qui défendaient l'art du maître quelques années auparavant ont jugé sévèrement son impact sur la jeune génération:

Il y a longtemps qu'on leur [aux artistes belges de la jeune génération] recommande de veiller sur eux, de ne pas sacrifier à la modernité, de ne pas s'évertuer à des imitations serviles qui tuent l'inspiration [...] si rien n'y fait, eh bien, alors c'est le naufrage de l'art belge. [...] [M]on patriotisme à moi crie aux Belges: l'ennemi est entré dans la maison, garde à vous $!^{40}$

De jeunes peintres comme Frantz Charlet et Théo van Rysselberghe, membres comme Frederic de L'Essor, ont ainsi été mis au banc des pasticheurs serviles de Bastien-Lepage. ${ }^{41}$ Frederic n'était pas exempt de ces critiques. Verhaeren soulignait concernant le triptyque La Légende de saint François que «la préoccupation française domine, dans le sujet, la façon de le concevoir et de le traiter, d'harmoniser les teintes plus distinguées que solides». ${ }^{42}$ Cette domination affaiblissait d'après l'auteur «la tradition flamande» et «l'originalité nationale». ${ }^{43}$ Il en est de même dans la description que fait Lemonnier des Marchands de craie qui «trahissait, sous une rare pénétration du détail physionomique 
44. Lemonnier, Histoire des Beaux-arts en Belgique, $2^{\mathrm{e}}$ ed., op. cit., 1887, p. 318-320.

45. Brogniez, op. cit, p. 87.

46. Ibid.

47. Jean-Paul Bouillon, «Le moment symboliste», Revue del'art, Paris, vol. 96, 1992, p. 1-7.

48. Anonyme, «La Huitième Exposition de l'Essor», L'Art moderne, Bruxelles, $4^{\mathrm{e}}$ année, $\mathrm{n}^{\circ} 2$, 13 janvier 1884, p. 12.

49. C'est à cette date que Frederic découvre, à l'occasion du mariage d'une proche, le village ardennais de Nafraiture. Cette petite localité reculée et isolée de toute ville devient avec ses habitants les motifs principaux de l'artiste. Il y passe plusieurs mois chaque année trente ans durant.

50. Gabriel P. Weisberg, Beyond Impressionism: The Naturalist Impulse in European Art 1860-1905, Londres, 1992, p. 218.

51. Paul Aron, «L'Expérience belge de l'art social», dans Neil McWilliam, Catherine Méneux et Julie Ramos (dir.), L'Art social en France. De la Révolution à la Grande Guerre, Rennes, 2014, p. 225-226. et une atténuation voulue des gammes coloristes, l'influence momentanée d'un artiste consacré en France par de retentissants succès, Bastien Lepage» et que le critique décrivait comme procédant d' «un réalisme de demi-caractère». Cette "passagère tendance» à la «distinction un peu superficielle» détournait «le bel instinct robuste de la race» et la «vision flamande des choses». ${ }^{44}$

Perçue comme stade ultime de la modernité par Solvay et dans un premier temps par Lemonnier, défendue par de nombreuses revues ou cercles artistiques comme L'Essor, la nouvelle école naturaliste a été favorablement accueillie dans sa définition même, la revendication d'un art réaliste amélioré par l'observation précise et scientiste de la nature et du quotidien. Cependant, la nouvelle suprématie française sur la scène internationale est venue perturber la réception positive de ce mouvement. En réaction au naturalisme international (trop français), les intellectuels belges dénoncèrent le manque de "picturalité» ${ }^{45}$ des œuvres étrangères, valeur intrinsèquement liée à l'art flamand et capable, selon eux, de contrer l'école française sans décrédibiliser l'orientation de la nouvelle école. L'art de Bastien-Lepage se retrouvait ainsi critiqué dans sa facture trop lisse, trop sèche, trop terne, a contrario de l'archétype de l'art flamand décrit par Taine puis Lemonnier: une peinture sensible à la matière dans laquelle prédomine la couleur sur le dessin. Plus qu'un mouvement spécifique (réalisme ou naturalisme), c'est le rapport à la réalité, ainsi qu'à la matérialité qui a conditionné le discours belge artistique puis littéraire. ${ }^{46}$

\section{Léon Frederic et le naturisme: I'identité flamande retrouvée}

La problématique identitaire constituait l'un des fondements de l'idéologie de l'intelligentsia belge. Dépassant les clivages, cette obsession se retrouvait aussi bien sous la plume de critiques conservateurs que dans les écrits des progressistes. Si l'emprunt de la voie naturaliste par la jeune génération d'artistes belges était loué et légitimé dans un premier temps par ces auteurs pour le caractère atavique d'une école artistique attirée par l'observation et la représentation de la réalité, l'adhésion d'une grande partie d'entre elles au «moment»» ${ }^{47}$ symboliste, célébrant le culte de l'Idée et non du réel, semblait beaucoup moins conciliable avec l'entreprise d'une histoire de l'art belge cohérente. La réception de l'œuvre de Léon Frederic, par ses rattachements aux deux tendances, livre un éclairage tout à fait intéressant.

L'art de Frederic connaît dès le début des années 1880 des tâtonnements stylistiques symptomatiques des recherches esthétiques de L'Essor et des querelles artistiques soulevées par la presse. Alternant des œuvres inscrites dans le sillage de Bastien-Lepage et du naturalisme international, comme son triptyque Les Marchands de craie, avec des œuvres d'un style plus personnel caractérisé par de larges touches et par «une coloration noirâtre, terne et triste», ${ }^{48}$ comme Les Femmes à loques (1883) ou La Vieille Servante (1884), Frederic poursuit inlassablement dans les deux cas la veine du naturalisme social en représentant la vie des pauvres gens des villes et du milieu rural et paysan dès $1883 .{ }^{49}$ Cette orientation se retrouve développée à la même époque par de nombreux écrivains belges comme Lemonnier ou Verhaeren, ${ }^{50}$ ainsi que dans le plaidoyer pour un art social exposé par Edmond Picard dans les pages de L'Art moderne. ${ }^{.1}$ Ces nombreuses œuvres consacrées à la vie des humbles 

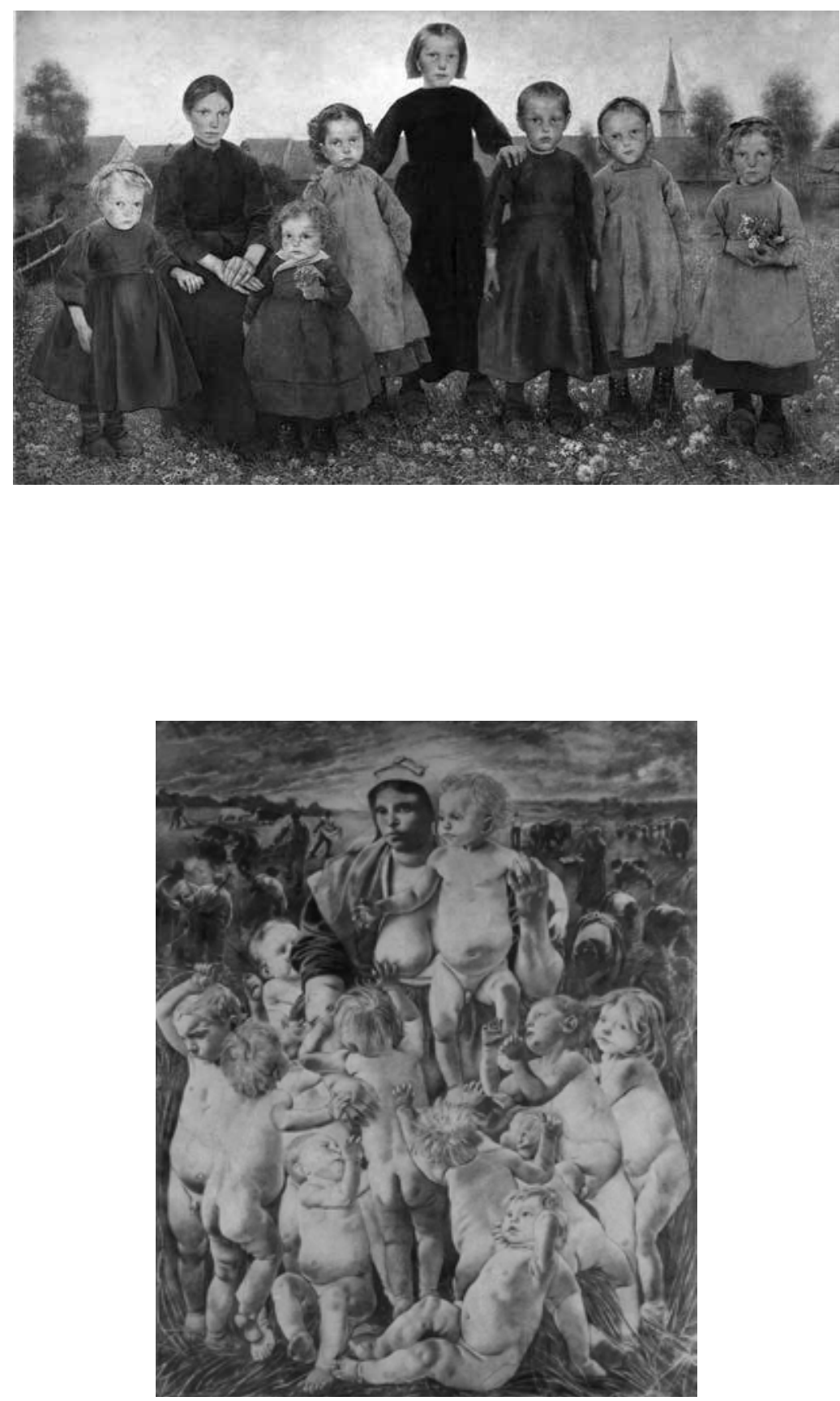

Figure 3. Léon Frederic, Les Boëchelles, 1886 , huile sur toile, $115 \times 202 \mathrm{~cm}$. Bruxelles, Musées royaux des Beaux-arts de Belgique (photo @ KIK-IRPA, Bruxelles, Belgique).
Figure 4. Léon Frederic, La Terre, 1888 , fusain, $184 \times 145 \mathrm{~cm}$. SaintPétersbourg, musée de l'Hermitage (photo @ KIK-IRPA, Bruxelles, Belgique). 
52. Anonyme, "L'Essor. viıl Exposition annuelle», L'Art moderne, Bruxelles, $4^{\mathrm{e}}$ année, $\mathrm{n}^{\circ} 2$, 13 janvier 1884 , p. 12.

53. Citons par exemple: Anonyme, «La Huitième Exposition de l'Essor», L'Art moderne, Bruxelles, 4 année, $n^{\circ} 2$, 13 janvier 1884, p. 12; Paul Mantz, «Le Salon (Champ-deMars) II", Le Temps, Paris, $31^{\mathrm{e}}$ année $\mathrm{n}^{\circ} 10998,28$ juin 1891, p. 1.

54. Abel Moran, «L'Essor», L'Elan, Liège, $2^{\mathrm{e}}$ année, 1886 , p. 24.

55. XXXIII Exposition triennale de Gand. Salon de 1886, Gand, 1886, p. 79. Fuvre à ne pas confondre avec Les Boëchelles (1888) du Musée royal des Beaux-arts d'Anvers.

56. Pierre Gervaix, «Le Salon de Gand", Journal des Beaux-arts et de la littérature, Bruxelles, $28^{\mathrm{e}}$ année, $\mathrm{n}^{\circ} 18$, 30 septembre 1886 , p. 138.

57. L'utilisation de la photographie comme aide à la préparation d'un tableau était très courante chez les artistes proches du courant naturaliste; voir Gabriel Weisberg (dir.), L'Illusion de la réalité. Peinture, photographie, théâtre et cinéma naturalistes, 1875-1918, catalogue d'exposition, Bruxelles, 2010, p. 31-43 Malgré les doutes que l'on peut avoir sur l'utilisation de ce médium par Frederic pour ce tableau, cette composition découle sans doute d'un désir de rivaliser avec la photographie et de la dépasser.

58. Le terme de Boëchelle signifie en vieux patois wallon «jeune fille». Il dénote la volonté de Frederic d'ancrer précisément son sujet dans une réalité observée et vécue.

59. Max Waller, «Chronique artistique: Le Salon de Bruxelles de 1887", La Jeune Belgique, Bruxelles, $7^{\mathrm{e}}$ année, $\mathrm{n}^{\circ} 10,12$ octobre 1887 , p. 324 .

6o. Lettre autographe signée de Léon Frederic à Firmin Baes, non datée. Archives de l'art contemporain de Belgique, Bruxelles: $n^{\circ} 83.741$. apportent à Frederic un succès unanime. Toutefois, les critiques remarquent la difficulté de l'artiste à se séparer de l'influence trop écrasante de l'art de Bastien-Lepage. Ainsi L'Art moderne met en garde «[c]elui des jeunes dont l'avenir est sur le point de se dessiner, qui est arrivé à la limite extrême où les promesses doivent se réaliser, sous peine de demeurer stériles [...]». ${ }^{52}$ L'ancrage par la critique des œuvres de Frederic dans la tradition de l'art belge, débarrassées de toutes influences étrangères, se fait au fil du temps de plus en plus précis. Ainsi, les critiques n'auront de cesse de rattacher Frederic à l'héritage des primitifs flamands ${ }^{53}$ ou alors emploieront des références nationales, comme le critique Abel Moran qui s'exclamera: «Cela est sain comme du Lemonnier». ${ }^{54}$ En 1886, Frederic expose au Salon de Gand pour la première fois ses Boëchelles, ${ }^{55}$ | fig. 3 | premier des cinq panneaux de ce qui deviendra quelques années plus tard la suite des Âges du paysan (1885-1887). Cette œuvre a été qualifiée de «coup de pistolet» par le critique d'art conservateur Pierre Gervaix. ${ }^{56}$ Le tableau représente huit petites paysannes vues de face dans des poses prises sur le vif, installées dans une prairie des environs du village ardennais de Nafraiture. Dans la pose frontale et l'alignement des modèles, la composition semble faire écho à la pratique photographique, tandis que la gaucherie des jeunes filles corrobore l'impression d'instant pris sur le vif.57 Cette suite de portraits de jeunes paysannes wallonnes, comme l'indique le titre donné par l'artiste, ${ }^{58}$ exprime les recherches du peintre autour du naturalisme-la représentation sans fard de petites gens observés directement dans leur milieu-mais également, d'après les critiques, sa prise de distance avec Bastien-Lepage. À partir de cette exposition, Frederic concentre tous ces efforts à portraiturer les paysans, peindre des paysages ardennais et réaliser de grandes fresques illustrant la vie des plus humbles, comme avec la suite monumentale composée de vingt-trois fusains, intitulée Le Lin et Le Blé (18871889), ou avec Les Âges du Paysan. Le poète belge Max Waller loue au sujet de cette dernière œuvre la capacité de Frederic à atteindre par la représentation de la «laide vérité» la poésie des gens de la glèbe qui «ont une vie d'animalité pensante qui empoigne jusqu'aux fibres».$^{59}$ Par cette œuvre, Frederic tente de dépasser la simple représentation mimétique de paysans ardennais observés en plein air pour livrer une représentation immuable des travailleurs de la glèbe. À cette époque, la réception de Frederic tourne à la consécration. Ces vastes synthèses louant la vie rurale et son rapport à la nature affirment les réflexions spirituelles et idéalistes qui seront désormais une constante dans l'œuvre du peintre. Le fusain allégorique représentant une Alma Mater | fig. $4 \mid$ sous les traits d'une paysanne allaitant ses enfants, les douze mois de l'année, en est un exemple. Ce panneau séparait les deux suites du Lin et du Blé, synthétisant les divers travaux rattachés à ces deux matières premières. Par cette allégorie centrale, Frederic inscrit la condition paysanne à «leur vie de nature», comme il l'écrira lui-même. ${ }^{60}$ Cette nouvelle tendance spirituelle et idéaliste trouve son origine dans le désir de l'artiste de dépasser la stricte représentation d'une réalité observée. La lecture de certaines revues qui ont développé et encouragé le mouvement symboliste en Belgique dès la fin des années 1880 , telles La Jeune Belgique ou La Basoche, n'est sûrement pas étrangère à cette nouvelle orientation. On retrouve l'ambiguïté de l'œuvre de Frederic chez de nombreux peintres de L'Essor, eux aussi réceptifs tant aux valeurs naturalistes que 


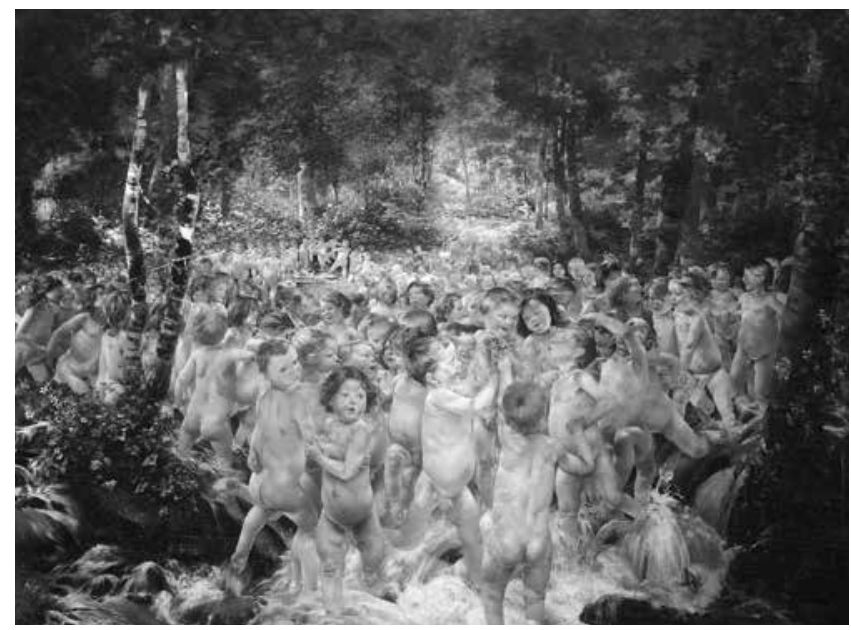

Figure 5. Léon Frederic, Le Ruisseau, huile sur toile, panneau central du triptyque Le Ruisseau, 18901899 , huile sur toile, $206 \times 282 \mathrm{~cm}$. Bruxelles, Musées royaux des Beauxarts de Belgique (photo () KIK-IRPA, Bruxelles, Belgique).

Figure 6 (ci-contre). Léon Frederic, Le Peuple verra un jour le lever du soleil, 1890-1891, huile sur toile, $149 \times 100 \mathrm{~cm}$ (panneau central); $106 \times 139 \mathrm{~cm}$ (panneaux latéraux). Propriété de l'État belge, œuvre gérée par la Fédération Wallonie-Bruxelles (photo @) Sylvain Jennebauffe).

symbolistes. C'est le cas de Jean Delville qui au Salon de L'Essor de 1888 expose une allégorie de La Terre représentée sous les traits d'un semeur. Mentionnons les premières œuvres de Constant Montald ou Émile Fabry, qui deviendront avec Delville les figures les plus importantes du symbolisme en Belgique. ${ }^{61}$ L'évolution de certains membres de L'Essor vers la tendance symboliste, mêlée à des désaccords internes, provoque la dissolution du cercle et la création au début de l'année 1892 de Pour l'Art. ${ }^{62}$ Si, à l'origine, ce cercle est créé dans l'optique d'une pluralité esthétique comme l'était L'Essor, très rapidement, sous l'impulsion de Delville et sous l'influence de Joséphin Péladan et de la Rose+Croix parisienne, certains de ses membres se sont inscrits dans une voie symboliste et ésotérique. Frederic choisit de ne pas adhérer à Pour l'Art, bien que certaines amitiés aient pu l'y pousser. Cependant, les œuvres de l'artiste s'orientent elles aussi vers le courant symboliste, comme le démontrent ses envois aux expositions parisiennes de la jeune Société Nationale des Beaux-Arts: citons La Nuit (1891) ou La Vanité des grandeurs (1893)... Cette tendance va s'exprimer de différentes façons au cours des années 1890 . Le peintre usera régulièrement du processus d'allégorisation, prenons pour exemple Le Ruisseau (1890-1899) | fig. 5 | ou La Pensée qui s'éveille (1891). Conjointement à ces toiles, Frederic présentera des œuvres d'un symbolisme simple clamant un discours utopique du retour de l'homme à la nature comme un retour vers un Eden primitif et illustrant sa sensibilité sociale, notamment dans son triptyque Le Peuple verra un jour le lever du soleil (1891). | fig. 6 |

Ce changement stylistique des années 1890 marque un tournant dans la carrière de Frederic. Le peintre jouissait à cette époque d'une reconnaissance internationale et nationale unanime, aboutissant à la publication d'une

61. Clerbois, op. cit., p. 23

62. Ibid., p. 59 .

63. Edmond-Louis de Taeye, Les Artistes belges contemporains, Bruxelles, 1894, p. 293-307.

64. Vandeputte, «Léon Frédéric», op. cit., p. 101-107. première biographie en $1894 .{ }^{63}$ Quelques mois plus tard, l'article monographique de Vandeputte, paru dans la nouvelle revue L'Art jeune qu'il fonde et dirige, est un témoignage de l'attention portée à l'artiste. ${ }^{64}$ Le programme de cet organe était à sa création assez évasif et se contentait de proclamer son soutien aux jeunes artistes, d'obédience non précisée, afin de «rajeunir l'Art» 


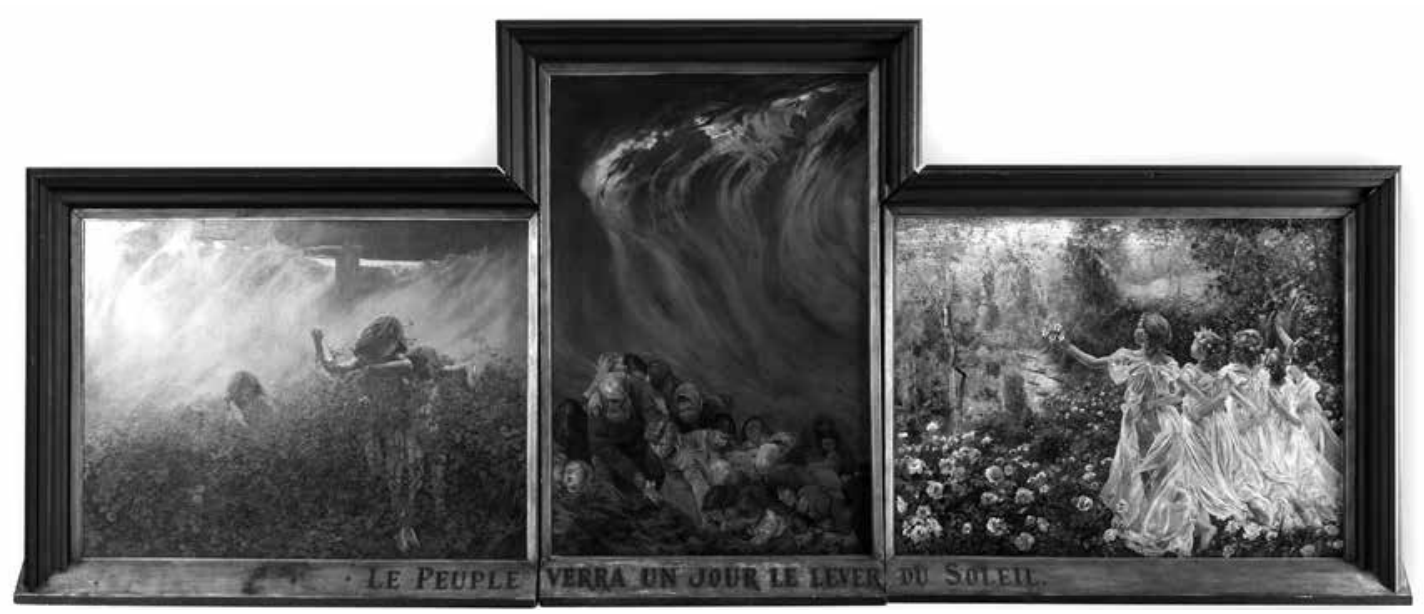

65. L'Artjeune, «Notre Programme», L'Art jeune, Bruxelles, 1 ère année, $\mathrm{n}^{\circ} 1,15$ janvier 1895 , p. 1.

66. Vandeputte, «Léon Frédéric», op. cit., p. 103.

67. Ibid., p. 102

68. Ibid., p. 107.

69. Ibid., p. 102.

70. Ibid., p. 103.

71. Baes, op. cit., p. 679

72. Vandeputte, «Léon Frédéric», op. cit., p. 106.

73. Saint-Georges de Bouhélier, "Un Manifeste», Le Figaro, Paris, $43^{\mathrm{e}}$ année, $\mathrm{n}^{\circ} 10,10$ janvier 1897, p. 4-5.

74. Le Blond, op. cit., 1896.

75. Ibid., p. 32. en les laissant «parler librement de leurs formes d'art, de leurs idées, de leurs salons, et tenir chacun en liberté pleine mais responsable [...]». ${ }^{65}$ Peu à peu, englobant l'éphémère revue Stella, L'Artjeune dépasse la voie d'un «naturalisme outré d'hier» et «l'Idéalisme réactionnaire passager» ${ }^{66}$ et se positionne dans le sillage d'un nouveau néologisme artistique: le naturisme. ${ }^{67}$ Vandeputte y définit son concept par le prisme de l'œuvre de Frederic, intronisé comme «le Premier des peintres belges, Artistes». ${ }^{68}$ Frederic devient ainsi l'un des chefs de file, malgré lui, d'un mouvement prônant l'«Art dans l'expression de la nature, de la vie, de l'homme en toute simplicité, en toute réalité». ${ }^{69}$ Pour Vandeputte, Frederic est l'artiste «qui a le mieux fixé cette formule en son œuvre», reflétant son intime personnalité de peintre "simple, sincère, amoureux de tout ce qui correspondait à sa nature: la sincérité des humbles, la simplicité des campagnes, et parfois les misères urbaines». ${ }^{70}$ L'auteur perçoit l'œuvre de Frederic comme relevant d'un "panthéisme idéo-réaliste» ${ }^{71}$ et glorifiant la représentation d'une humanité archétypale et éternelle intimement liée à la nature. Cette observation trouve son paroxysme dans la suite des Âges du paysan:

C'est, infiniment simple et naïve, une longue suite d'êtres primitifs, graduant les âges divers de la vie terrienne; mais cela est vrai et vécu, c'est la nature toute simple et très belle des paysans quelconques dont émane l'inaltérable arome de poésie qui se dégage semblablement de la terre, des moissons et des bœufs, de toute la nature. ${ }^{72}$

Le naturisme connaît un succès certain en Belgique auprès de la jeune génération, mais également en France. Deux écrivains, Saint-Georges de Bouhélier et Maurice Le Blond, en sont les principaux protagonistes parisiens. Le premier en publie le manifeste dans les colonnes du Figaro en $1897,{ }^{73}$ et le second s'impose comme son principal défenseur et propagateur, notamment par la publication de son Essai sur le Naturisme. ${ }^{74}$ L'année 1895 marque l'apparition de ce néologisme en Belgique, et il sera rapidement relayé en France. Ainsi Le Blond exhorte dès 1896 les artistes à suivre «une doctrine plus simple et naïve, vers un art d'humanité que nous pourrons appeler-avec un jeune écrivain belge, $M$. Van de Putte-Le Naturisme». ${ }^{75}$ La création à Paris de la revue 
76. Henri Vandeputte,

"Choses», L'Art jeune, Bruxelles, $2^{\mathrm{e}}$ année, $n^{\circ} 2,15$ février 1896, p. 65.

77. Maurice Le Blond, «Documents sur la poésie contemporaine», La Plume, Paris, $n^{\circ} 205,1^{\text {er }}$ novembre 1897 , p. 658.

78. Edgar Baes, «Le Paysage du naturisme», La Plume, Paris, $n^{\circ} 205$,

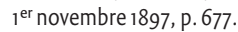

79. Maurice Le Blond, Essaisurle Naturisme, Paris, 1896, p. 119.

80. Henri Vandeputte, «Nos Artistes. Firmin Baes», L'Art jeune, Bruxelles, $1^{\text {ère }}$ année, $n^{\circ} 11-12,15$ décembre 1895, p. 259-261.

81. Le Blond, op. cit., 1897, p. 658 .

82. Ibid.

83. Citons par exemple les ouvrages de Clerbois, op. cit.; ou Michel Draguet, Le Symbolismeen Belgique, Bruxelles, 2010.

84. Jean-David Jumeau Lafond, Les Peintres de l'âme: le symbolisme idéaliste en France, catalogue d'exposition, Gand/Anvers, 1999.

85. Vandeputte, «Léon Frédéric», op. cit., 1895, p. 105.
L'Âme Nouvelle, ${ }^{76}$ dont le comité aurait été constitué entre autres de Bouhélier et Vandeputte, devait sceller dès 1896 cette alliance belgo-française dans la défense commune de ce mouvement, avant son abandon pour des raisons inconnues. Le naturisme s'affirme, aussi bien en Belgique qu'en France, comme une continuation du naturalisme, en prônant un retour à l'observation de la nature. ${ }^{77} \mathrm{Il}$ en est même, d'après l'historien belge Edgar Baes, le "comble». ${ }^{78}$ Cependant, le courant naturiste s'évertue à se distinguer

[...] en ce qu'à l'observation il préfère l'émotion. Sacrifiant la documentation exacte, il estime davantage les sites éternels. Il est moins pittoresque, mais plus sublime et néglige les individus pour les archétypes. Ainsi il peut créer des héros véridiques et atteindre en même temps, à l'Épopée. ${ }^{79}$

L'Art Jeune s'emploie à défendre une jeunesse artistique s'inscrivant dans une voie naturiste dont Frederic est désigné comme le parangon. L'exemple du peintre Firmin Baes est frappant. Élève de Frederic depuis le début des années 189o, Baes se voit gratifier d'un court article monographique écrit par Vandeputte alors que le jeune peintre était «ignoré du public, parce qu'il n'a[vait] jamais exposé». ${ }^{80}$ Ce zèle dont fait preuve le critique s'explique notamment par le combat idéologique dans lequel s'est engagée cette jeune garde dont il fallait gonfler les rangs et affirmer l'actualité contre le symbolisme. Jugé comme «réactionnaire» par Le Blond, le symbolisme marquait, selon les écrivains naturistes, «un arrêt dans l'évolution intellectuelle [...] en voulant réagir contre l'Encyclopédie, le mouvement scientifique, la pensée positiviste et l'effort du naturalisme». ${ }^{81}$ Poursuivant dans l'invective, Le Blond affirme que "c'est à la marche même de l'humanité qu'il [le symbolisme] prétendait s'opposer». ${ }^{82}$

Le symbolisme a eu un impact considérable en Belgique autant dans les œuvres littéraires que plastiques. ${ }^{83}$ L'un de ses principaux représentants était le peintre Delville, fondateur des Salons idéalistes bruxellois et propagateur de la doctrine esthétique, ésotérique et rosicrucienne de Péladan parmi les jeunes générations. ${ }^{84}$ Difficilement classifiable, l'œuvre de Frederic a souvent été rattaché par certains points à ce mouvement:

On a beaucoup discuté sur le symbolisme de Frédéric. D'aucuns l'ont jugé inférieur à son réalisme, d'autres l'ont démoli ou applaudi, beaucoup même l'ont nié. [...] Son symbolisme, pour autant qu'il y en ait, car ses tableaux, même les récents, sont plutôt allégoriques, son symbolisme est de toute simplicité. Il se comprend à première vue. ${ }^{85}$

La minimisation du penchant symboliste de l'artiste n'est pas une surprise venant de l'un des fondateurs du naturisme, mouvement qui, comme nous l'avons vu, souhaitait se positionner contre les symbolistes. L'entrisme dont faisaient preuve les propagateurs français de cette esthétique en Belgique, notamment l'autoproclamé Sâr Mérodack Joséphin Péladan, et leur influence provoquèrent une réaction épidermique chez une grande partie de la scène intellectuelle belge, comme en témoigne la critique du peintre James Ensor face à la nomination de Jean Delville au Prix de Rome en 1895. Dénonçant les «contorsions épileptiques» du nouveau lauréat, Ensor signale le changement stylistique brutal de Delville, passant d'une peinture réaliste à une œuvre idéaliste découlant du discours du «trop français» Sâr Péladan, initiateur d'une italianisation reniant les «qualités flamandes». Ensor continue: 
86. James Ensor, «Le prix de Rome», Le Cog rouge, Bruxelles, $1^{\text {ère }}$ année, $n^{\circ} 8$ et 9 , décembre 1895-janvier 1896, p. 439-440.

87. Gustave Vanzype, Léon Frederic, Bruges, 1942, p. 54.

88. Gustave Vanzype, Eugène Laermans, Bruxelles, 1908, p. 2.

89. Bouhélier, op. cit., p. 4. nous voyons sans étonnement le peintre de La Terre et de l'Accouchée baiser la robe des vierges Botticelliennes, s'attacher à la queue des mages [...]. Certes, les plaisants symbolistes se gorgent de fiente Botticellienne. [...] Personnages boursouflés, négation absolue de coloris, manque complet de qualités flamandes, etc., etc. ${ }^{86}$

À côté du discours de certains artistes, l'histoire de l'art établie au tournant du $\mathrm{xx}$ siècle a tenté elle aussi de légitimer le rejet du symbolisme et de l'ostraciser par le prisme du nationalisme. L'ouvrage de Camille Lemonnier intitulé L'École belge de peinture 1830-1905, paru en 1906, en est l'exemple le plus probant et s'inscrit entièrement dans la continuité de ses ouvrages à caractère nationaliste publiés quelques années auparavant, opposant un art réaliste et flamand, donc national, avec un art symboliste, français et latin, donc étranger au terreau culturel de la Belgique. Pourtant, la réalité de la scène artistique tournée vers le symbolisme a sûrement joué contre cette ostracisation systématique. Certains historiens ont souhaité minimiser l'impact de cette mouvance sur l'art des peintres belges en parlant d'errance momentanée; c'est le cas de Gustave Vanzype qui pour parler de l'art de Frederic signale ces «défaites_ces demi-défaites» ou des «œuvres confuses» quand «il lui arriva [...] de trop s'éloigner de la réalité». ${ }^{87}$ Quant aux peintres indissociables du mouvement symboliste, ils sont balayés d'un revers de main comme les tenants d'un art «accessible seulement à quelques uns» et qui n'a aucun lien avec la tradition nationale. ${ }^{88}$

Conjointement à ce discours excluant, une autre histoire de l'art orientée vers l'assimilation du symbolisme est instaurée. Le naturisme semble, en Belgique, prendre toute sa place dans ce processus, et ceci ne paraît pas étonnant en sachant que le sentiment nationaliste se révélait être le centre névralgique du manifeste de Bouhélier. S'appuyant sur les travaux de Taine, l'auteur prône «la résurrection des cultes nationaux [...] débarass[és] de cette pensée étrangère qui encombre encore notre esprit [français/latin] [et qui] défigure l'esprit de la race». ${ }^{89}$ Nous comprenons aisément que cette facette ne pouvait que séduire l'intelligentsia belge en quête d'une identité artistique, surtout en

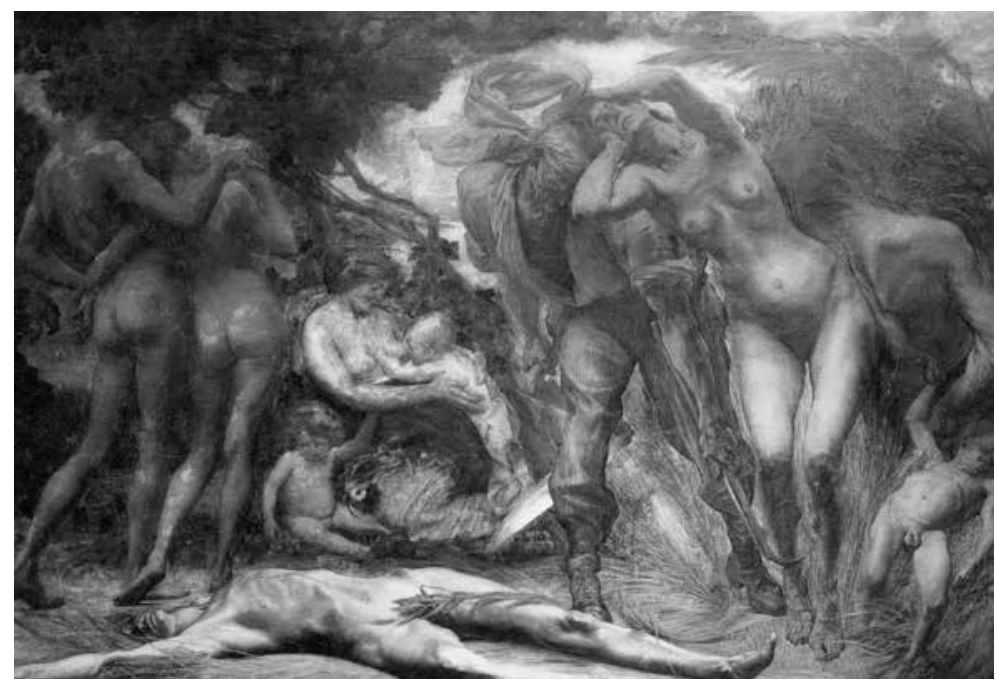

Figure 7. Auguste Levêque, LeTriomphe de la mort, 1905, huile sur toile, $199 \times 291 \mathrm{~cm}$. Anvers, Musée royal des Beaux-arts (inv. 9.038/1-3; photo @ KIK-IRPA, Bruxelles, Belgique). 
90. Baes, op. cit., p. 678 .

91. Ibid.

92. De Taeye, op. cit., p. 303

93. Clerbois, op. cit., p. 15152.

94. Camille Lemonnier, «Introduction», LaPlume, Paris, $\mathrm{n}^{\circ} 205,1^{\mathrm{er}}$ novembre 1897, p. 650. tenant compte du fait que le naturisme louait le culte de la nature et l'observation de la réalité. Encourageant la poursuite d'une histoire de l'art nationale minimisant un naturalisme en crise et un symbolisme définitivement trop latin, les initiateurs de l'histoire de l'art belge ont trouvé dans le naturisme le fer de lance de leur idéologie capable de réunir des tendances artistiques aussi diverses que contradictoires. C'est Jules Du Jardin qui, dans son ouvrage L'Art flamand, paru en 1896, poursuit cette possible symbiose du symbolisme avec le ferment artistique national. L'auteur tente de penser le symbolisme en rapport avec la tradition des Primitifs flamands. Il fonde cette interprétation sur plusieurs travaux, notamment ceux d'Edgar Baes, L'Allégorie et le Symbole, lauréat d'un concours à l'Académie royale en 1899. Baes, disciple du naturisme, glorifie l'allégorie comme origine de l'harmonie entre symbolisme et réalisme, tentant de défendre ainsi un art plus sensible et moins hermétique. Quelques années auparavant, le même auteur, dans un numéro spécial de la revue française La Plume, clamait haut et fort le rôle que pouvait jouer ce mouvement en Belgique: «Dans notre école de coloris réaliste, les essais de peinture symboliste n'ont eu aucune chance de succès jusqu'ici. [...] Ici intervient le rôle du Naturisme». ${ }^{90}$ Et il concluait son article d'un souhait non moins explicite:

[...] le panthéisme est tellement l'essence même de notre sentiment d'art naturaliste que nous considérions comme un crime de ne pas attirer l'attention de tous nos artistes sur cette formule qui sort sereine et triomphante des tâtonnements et du chaos d'an$\tan$. Notre art atavique [...] retrouverait ce que nous aspirions à voir éclater de nouveau au grand jour, la sublime inspiration de la réalité transfigurée! ${ }^{91}$

Edmond-Louis de Taeye, le premier biographe de Frederic, corrobore cette lecture lorsqu'il proclame que «[c]'est même dans ce contraste entre l'idéal qui fait songer au ciel, et le réel qui rappelle la terre, que réside l'attrait le plus vif des œuvres de l'artiste. D'autre part, ce mysticisme étrange est parfaitement flamand». ${ }^{92}$ De nombreux artistes tels les peintres Eugène Laermans, Auguste Levêque, Emile Fabry, Firmin Baes, ou le sculpteur Jef Lambeaux ont étayé par leurs œuvres et leurs carrières ce propos, ou se sont inscrits euxmêmes à rebours dans ce sillage. La conversion naturiste de Levêque en est un exemple probant, notamment par son usage récurrent de l'allégorie et la réflexion qu'il mena autour de cette forme de représentation après ses participations à L'Art jeune. ${ }^{93}$ On peut remarquer ce changement dans Le Triomphe de la mort, | fig. 7| qui reprend une iconographie proche de celle développée par Frederic, par exemple le thème de la moisson et l'inscription allégorique dans le monde rural.

S'émouvant de «leurs [celles des naturistes] croisades pour l'idée et la vie» et se targuant d'appartenir «à la même famille intellectuelle», ${ }^{94}$ Camille Lemonnier, grand instigateur du culte national dans les arts, marque sa conversion au naturisme dans les colonnes du numéro spécial de La Plume. Cette adhésion est bien une preuve de la persistance du discours nationaliste dans la définition des nouveaux courants artistiques en Belgique afin de créer une histoire de l'art belge cohérente. Ainsi, nous avons pu appréhender à travers la réception de l'œuvre de Frederic le sens idéologique et politique qui se cache derrière l'apparition des termes artistiques au sein d'une Belgique encline aux crises identitaires. 